\section{International Scientific Journal Theoretical \& Applied Science}

\author{
Vasiliy Evgenievich Polyakov \\ Senior Lecturer, \\ Department of Economic Analysis \\ Kuban State Agrarian University, \\ Krasnodar, Russia \\ Polyakov.VE@gmail.com
}

SECTION 31. Economic research, finance, innovation, risk management.

\title{
ECONOMIC ANALYSIS AND ASSESSMENT OF THE EFFECTIVENESS OF INVESTMENT PROJECTS ON THE BASIS OF DISCOUNTED PAYBACK PERIOD USING MICROSOFT EXCEL
}

\begin{abstract}
Defined the essence and the criteria for assessing the efficiency of investment projects using the discounted payback period. Proposed the method of calculating this index in Microsoft Excel. The analysis and assessment of the efficiency of the investment project using the discounted payback period.

Key words: analysis, economic efficiency, investment project, cash flow, discounting, payback period.

Language: Russian

Citation: Polyakov VE (2016) ECONOMIC ANALYSIS AND ASSESSMENT OF THE EFFECTIVENESS OF INVESTMENT PROJECTS ON THE BASIS OF DISCOUNTED PAYBACK PERIOD USING MICROSOFT EXCEL. ISJ Theoretical \& Applied Science, 11 (43): 21-26.

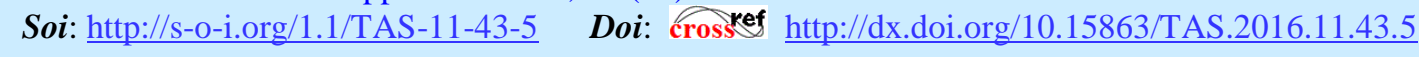

\section{ЭКОНОМИЧЕСКИЙ АНАЛИЗ И ОЦЕНКА ЭФФЕКТИВНОСТИ ИНВЕСТИЦИОННЫХ ПРОЕКТОВ НА ОСНОВЕ ДИСКОНТИРОВАННОГО ПЕРИОДА ОКУПАЕМОСТИ С ПОМОЩЬЮ МІСROSOFT ЕХCEL}

Аннотация: Определена сущзность и критерии оценки эффективности инвестиционных проектов на основе дисконтированного периода окупаемости. Предложена методика расчета этого показателя в Microsoft Excel. Проведен анализ и оценка эффективности инвестициооного проекта с помощью дисконтированного периода окупаемости.

Ключевые слова: анализ, экономическая эффективность, инвестиционный проект, денежный поток, дисконтирование, период окупаемости.
\end{abstract}

\section{Introduction}

Эффективность инвестиционного проекта характеризуется системой показателей, отражающих соотношение затрат и результатов применительно к интересам его участников.

В основе аналитического обоснования процесса принятия управленческих решений инвестиционного характера лежат оценка и сравнение объема предполагаемых инвестиций и будущих денежных поступлений. Поскольку сравниваемые показатели относятся к различным моментам времени, ключевой здесь является проблема их сопоставимости [3].

Сопоставимость разновременных денежных потоков инвестиционного проекта обеспечивается путем их дисконтирования, т. е. приведения к начальному (нулевому) этапу инвестирования. После приведения доходов и расходов инвестиционного проекта к одному периоду времени их можно сравнивать и оценивать эффективность инвестиций.

\section{Materials and Methods}

Одним из наиболее популярных критериев оценки эффективности инвестиционных проектов является срок их окупаемости.

Срок окупаемости инвестиционного проекта представляет собой период времени от начала его финансирования до момента, когда накопленная сумма доходов сравняется с амортизационными отчислениями и затратами по проекту.

В практике инвестиционного анализа сложилось два метода расчета срока окупаемости:

1) срок окупаемости на основе учетных оценок (Payback Period - PP);

2) срок окупаемости на основе

ISPC Education and Innovation, 
дисконтированных оценок (Discounted Payback Period - DPP) [2].

Первый - не предполагает временной оценки денежных поступлений, т. е. не учитывает разницу во времени возникновения расходов и получения доходов по инвестиционному проекту. Вследствие этого он предельно прост в расчетах, но дает очень приближенный (неточный) результат.

Второй - используется для нахождения уточненного срока окупаемости инвестиционного проекта с учетом временной оценки денежных потоков по нему. Вследствие этого он довольно трудоемок в расчетах, но дает относительно точный результат.

При сравнении сроков окупаемости по методу PP и DPP, всегда верно соотношение DPP $>$ PР. Таким образом, отсутствие временной оценки денежных потоков приводит к занижению реального срока окупаемости [1].

Расчет дисконтированного срока окупаемости производится путем решения уравнения, левая часть которого представлена суммой дисконтированных доходов по проекту, а правая - суммой дисконтированных расходов по нему. Если выразить это математически, то DPP $=\min n$ при котором:

$$
\sum \frac{S_{n}}{(1+i)^{n}}=\sum \frac{I_{n}}{(1+i)^{n}},
$$

где $S_{n}$ - доход от проекта за $n$ период, руб.;

$I_{n}$ - расход по проекту за $n$ период, руб.;

$\mathrm{n}$ - срок проекта, лет;

i - ставка дисконтирования, выраженная десятичной дробью [1].

Оценка инвестиционных проектов по критерию DPP может осуществляться двояко: а) проект принимается, если окупаемость существует;

б) проект принимается только в том случае, если срок окупаемости не превышает предельного срока, установленного инвестором.

Как уже говорилось, основным недостатком дисконтированного срока окупаемости инвестиционных проектов является сложность его расчета. Однако этот недостаток легко устранить, используя современные программные продукты для проведения инвестиционного анализа. Одним из наиболее распространенных из них можно считать Microsoft Excel. К сожалению, в Microsoft Excel не предусмотрено специальной функции для расчета срока окупаемости инвестиций. Однако его несложно осуществить с помощью составления соответствующих формул «вручную» [1].

Рассмотрим конкретный пример. Компания планирует приобрести новое оборудование стоимостью 7000 тыс. руб. и сроком эксплуатации 5 лет. От использования оборудования она будет получать дополнительный денежный приток в размере 2500 тыс. руб. ежегодно. Известно, что на третьем году эксплуатации оборудованию потребуется плановый ремонт стоимостью 300 тыс. руб. Необходимо обосновать целесообразность приобретения оборудования, если стоимость капитала по проекту составляет $20 \%$.

Представим исходные данные в виде таблицы. Для этого обозначим первую ячейку столбца $A$ как «Период» и заполним его порядковыми номерами лет реализации проекта (от 0 , который обозначает текущий (начальный) момент времени, до 5), а в столбце $B$ «Денежный поток»- укажем соответствующие величины доходов или расходов (рисунок 1).

\begin{tabular}{|c|c|c|c|c|}
\hline \multicolumn{3}{|c|}{ G15 } & $f_{x}$ & \multirow[b]{2}{*}{ D } \\
\hline 4 & A & B & C & \\
\hline 1 & Год & Денежный поток & $\begin{array}{c}\text { Дисконтированный } \\
\text { денежный поток }\end{array}$ & $\begin{array}{c}\text { Накопленный } \\
\text { дисконтированный } \\
\text { денежный поток }\end{array}$ \\
\hline 2 & 0 & -7000 & & \\
\hline 3 & 1 & 2500 & & \\
\hline 4 & 2 & 2500 & & \\
\hline 5 & 3 & 2200 & & \\
\hline 6 & 4 & 2500 & & \\
\hline 7 & 5 & 2500 & & \\
\hline \multicolumn{5}{|l|}{8} \\
\hline 9 & \multicolumn{2}{|c|}{ Стоимость капитала } & $20 \%$ & \\
\hline \multicolumn{5}{|l|}{10} \\
\hline 11 & \multirow{2}{*}{\multicolumn{2}{|c|}{$\begin{array}{l}\text { Дисконтированный период } \\
\text { окупаемости (DPP), лет }\end{array}$}} & & \\
\hline 12 & & & & \\
\hline \multicolumn{5}{|l|}{13} \\
\hline 14 & 11 & Лист4 Лист5 & Лист7 行 4 & $\rightarrow \square$ \\
\hline
\end{tabular}

Рисунок 1 - Пример таблицы для расчета DPP. 
Затем необходимо привести значения денежных потоков к текущему моменту времени, т. е. дисконтировать их на соответствующий период. Для этого добавим еще один столбец и назовем его «Дисконтированный денежный поток».

Для дисконтирования значений денежного потока можно воспользоваться функцией ПС, т. к. нам на основе известного будущего значения денежного потока (через год, два, три и т. д.) необходимо определить его приведенное (текущее) значение.

$\mathrm{B}$ ячейке $C 2$, соответствующей «нулевому» периоду, вызовем функцию ПС и заполним ее следующим образом (рисунок 2).

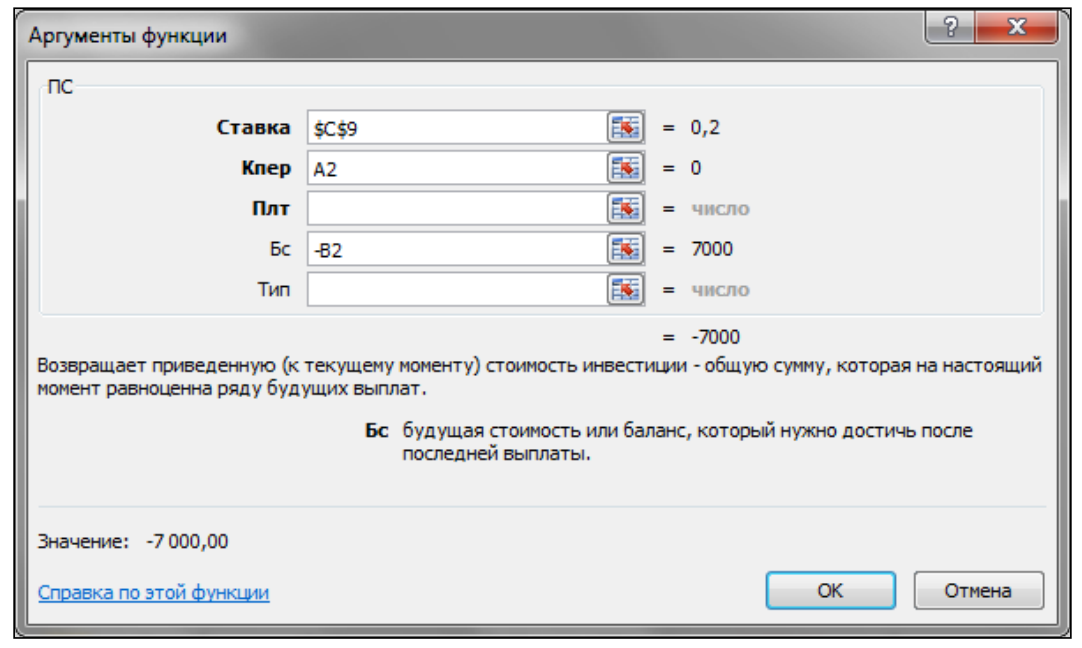

Рисунок 2 - Аргументы функции ПС.

Чтобы при «протягивании» полученной формулы значение аргумента «Ставка» не изменялось, преобразуем адрес ячейки C9 в абсолютный вид, нажав клавишу F4. В качестве аргумента Кпер используем порядковый номер года (ячейку A2). Так как при «протягивании» значение аргумента должно меняться, то оставим имя ячейки без преобразования в абсолютный вид. Таким образом, доход, полученный в первом году, будет дисконтирован на один год, во втором - на два года и т. д. Будущее значение денежного потока известно и содержится в ячейке $B 2$. Поскольку необходимо сохранить знак денежного потока, а функция ПС изменит его на противоположный, то укажем аргумент Бс с знаком «-». Значения денежного потока также должны изменяться от периода к периоду, поэтому оставляем имя ячейки в относительном виде.
Следует пояснить, что логика функции ПС такова: если указать аргумент Бс со знаком «+ », то Microsoft Excel будет полагать, что для того, чтобы в будущем получить некоторую сумму, нужно сейчас вложить средства и вернет значение со знаком « - ». И наоборот, если аргумент Бс со знаком «-», то Microsoft Excel полагает, что в будущем необходимо вернуть какую-то сумму, полученную сейчас, поэтому вернет положительное значение функции ПС. Таким образом, знаки аргумента Бс и значения функции ПС противоположны. В нашем примере этого необходимо избежать и мы заранее указываем обратный знак аргумента Бс.

После заполнения ячейки $C 2$ левой кнопкой мыши щелкаем на черный квадратик в нижнем правом углу ячейки и протягиваем на ячейки C3C7, автоматически заполняя их введенной формулой. 


\begin{tabular}{|c|c|c|c|c|c|c|}
\hline Impact Factor: & $\begin{array}{l}\text { ISRA (India) } \\
\text { ISI (Dubai, UAE } \\
\text { GIF (Australia) } \\
\text { JIF }\end{array}$ & $\begin{array}{l}=1.344 \\
=0.829 \\
=0.564 \\
=1.500\end{array}$ & $\begin{array}{l}\text { SIS (USA) } \\
\text { PИHЦ (Russia } \\
\text { ESJI (KZ) } \\
\text { SJIF (Morocco }\end{array}$ & $\begin{array}{l}=0.912 \\
=0.234 \\
=1.042 \\
=\mathbf{2 . 0 3 1}\end{array}$ & $\begin{array}{l}\text { ICV (Poland) } \\
\text { PIF (India) } \\
\text { IBI (India) }\end{array}$ & $\begin{array}{l}=6.630 \\
=1.940 \\
=4.260\end{array}$ \\
\hline
\end{tabular}

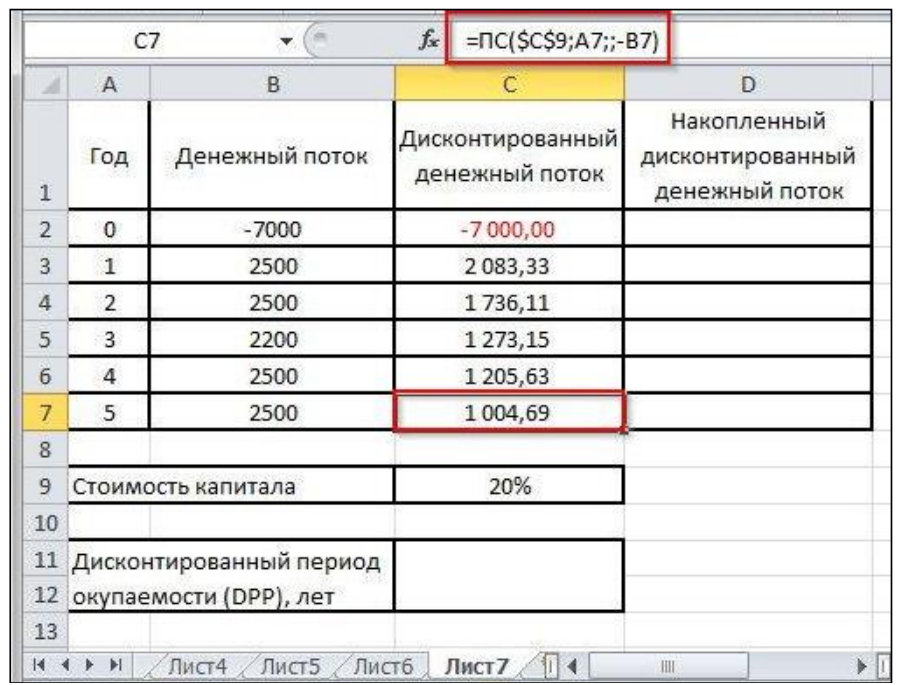

Рисунок 3 - Расчет дисконтированного денежного потока.

Затем необходимо за каждый период определить общую величину дисконтированных доходов (расходов) накопительным итогом. Для этого добавим еще один столбец и назовем его «Накопленный дисконтированный денежный поток».

Поскольку в «нулевом» периоде были осуществлены только затраты на приобретение оборудования (доходов не было), то приравняем значения ячеек $D 2$ и $C 2$, введя в ячейку $D 2$ формулу «=C2».

За первый год эксплуатации оборудования был получен доход в размере 2500 тыс. руб. (в текущих ценах 2083,33 тыс. руб.). Этот доход частично уменьшает затраты по проекту (7 млн. руб.) и на конец первого года непокрытыми остаются затраты в размере $7000-2083,33=$ 4916,67 тыс. руб.
Чтобы получить это значение, введем в ячейку $D 3$ формулу «=D2+C3» и поскольку в последующих периодах расчет аналогичен, то протянем ее на ячейки $D 4-D 7$ (рисунок 4).

Как мы видим, к концу четвертого года проект еще не окупился и величина непокрытого убытка составила 701,77 тыс. руб.

Первое положительное значение в столбце $D$ (302,92 тыс. руб.) появляется на пятом году эксплуатации оборудования и означает, что проект принес прибыль и все затраты окупились. Таким образом, период окупаемости проекта составляет от 4 (непокрытый убыток) до 5 (получена прибыль) лет.

Если доходы в течение года поступают равномерно, можно определить за какую часть пятого года окупился проект.

\begin{tabular}{|c|c|c|c|c|c|}
\hline \multicolumn{3}{|c|}{ D7 } & $f_{x}$ & $=\mathrm{D} 6+\mathrm{C} 7$ & \\
\hline \multirow[t]{2}{*}{4} & A & B & & C & D \\
\hline & Год & Денежный поток & \multicolumn{2}{|c|}{$\begin{array}{c}\text { Дисконтированный } \\
\text { денежный поток }\end{array}$} & $\begin{array}{c}\text { Накопленный } \\
\text { дисконтированный } \\
\text { денежный поток }\end{array}$ \\
\hline 2 & 0 & -7000 & & $-7000,00$ & $-7000,00$ \\
\hline 3 & 1 & 2500 & & 2083,33 & $-4916,67$ \\
\hline 4 & 2 & 2500 & & 1736,11 & $-3180,56$ \\
\hline 5 & 3 & 2200 & & 1273,15 & $-1907,41$ \\
\hline 6 & 4 & 2500 & & 1205,63 & $-701,77$ \\
\hline 7 & 5 & 2500 & & 1004,69 & 302,92 \\
\hline \multicolumn{6}{|l|}{8} \\
\hline 9 & \multicolumn{2}{|c|}{ Стоимость капитала } & & $20 \%$ & \\
\hline \multicolumn{6}{|c|}{10} \\
\hline$\frac{11}{12}$ & \multicolumn{2}{|c|}{$\begin{array}{l}11 \text { Дисконтированныи период } \\
12 \text { окупаемости (DPP), лет }\end{array}$} & & & \\
\hline \multicolumn{6}{|l|}{13} \\
\hline 144 & 11 & Лист5 Листе & & 80.14 & IIIII \\
\hline
\end{tabular}

Рисунок 4 - Расчет накопленного дисконтированного денежного потока

ISPC Education and Innovation, 


\section{Impact Factor:}

\begin{tabular}{|c|c|c|}
\hline SRA (India) & $=1.344$ & SIS (USA) \\
\hline ISI (Dubai, UAE & 0.829 & РИНЦ $($ Russia $)=0.234$ \\
\hline IF (Australia) & $=0.56$ & ESJI $(\mathrm{KZ})$ \\
\hline & $=1.500$ & SJIF $($ Morocco $)=\mathbf{2}$ \\
\hline
\end{tabular}

Из полученных данных следует, что на начало пятого года по проекту был непокрытый убыток в размере 701,77 тыс. руб., а за год получено 1004,69 тыс. руб. дохода (значение ячейки C7). Составив пропорцию, определяем, что проект окупился за $\frac{701,77}{1004,69}=0,7$ или $0,7 \times 12$
$=8$ месяцев пятого года. Таким образом, общий срок окупаемости проекта составляет 4,7 года или 4 года и 8 месяцев.

Произведем соответствующие расчеты в Microsoft Excel. Для этого, например, в ячейке $C 11$ введем формулу «=A6-D6/C7» (рисунок 5).

\begin{tabular}{|c|c|c|c|c|c|}
\hline \multicolumn{3}{|c|}{$\mathrm{C} 11$} & $f_{x}$ & \multicolumn{2}{|l|}{$=\mathrm{A} 6-\mathrm{D} 6 / \mathrm{C} 7$} \\
\hline 4 & A & B & & C & D \\
\hline 1 & Год & Денежный поток & \multicolumn{2}{|c|}{$\begin{array}{c}\text { Дисконтированный } \\
\text { денежный поток }\end{array}$} & $\begin{array}{c}\text { Накопленный } \\
\text { дисконтированный } \\
\text { денежный поток }\end{array}$ \\
\hline 2 & 0 & -7000 & & $-7000,00$ & $-7000,00$ \\
\hline 3 & 1 & 2500 & & 2083,33 & $-4916,67$ \\
\hline 4 & 2 & 2500 & & 1736,11 & $-3180,56$ \\
\hline 5 & 3 & 2200 & & 1273,15 & $-1907,41$ \\
\hline 6 & 4 & 2500 & & 1205,63 & $-701,77$ \\
\hline 7 & 5 & 2500 & & 1004,69 & 302,92 \\
\hline \multicolumn{6}{|l|}{8} \\
\hline 9 & \multicolumn{2}{|c|}{ Стоимость капитала } & & $20 \%$ & \\
\hline \multicolumn{6}{|l|}{10} \\
\hline 11 & \multirow{2}{*}{\multicolumn{2}{|c|}{$\begin{array}{l}\text { Дисконтированный период } \\
\text { окупаемости (DPP), лет }\end{array}$}} & & & \\
\hline 12 & & & & 4,70 & \\
\hline \multicolumn{6}{|l|}{13} \\
\hline 14 & 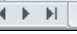 & Іист $4 /$ Лист5 / Лист6 & & ICT7 हूत & IIIII \\
\hline
\end{tabular}

Рисунок 5 - Расчет дисконтированного периода окупаемости.

Первая часть этой формулы «=A6...»- это целое количество лет за которое окупился проект (4 года), а вторая часть «...D6/C7» - это дробная часть следующего (пятого) года, составляющая полный срок окупаемости. Между этими частями стоит не знак « + », а знак « - », чтобы компенсировать знак «- » ячейки D4 (минус на минус, как известно, дает плюс).

В конечном итоге ячейки в данном примере должны быть заполнены следующим образом (рисунок 6).

\begin{tabular}{|c|c|c|c|c|c|}
\hline \multicolumn{3}{|c|}{ F14 } & $\left(-f_{x}\right.$ & & \\
\hline 4 & A & B & C & D & \\
\hline 1 & Год & $\begin{array}{c}\text { Денежный } \\
\text { поток }\end{array}$ & $\begin{array}{c}\text { Дисконтированный } \\
\text { денежный поток }\end{array}$ & $\begin{array}{c}\text { Накопленный } \\
\text { дисконтированный } \\
\text { денежный поток }\end{array}$ & \\
\hline 2 & 0 & -7000 & $=\Pi C(\$ C \$ 9 ; A 2 ; ;-B 2)$ & $=\mathrm{C} 2$ & \\
\hline 3 & 1 & 2500 & $=\Pi C(\$ C \$ 9 ; A 3 ; ;-B 3)$ & $=\mathrm{D} 2+\mathrm{C} 3$ & \\
\hline 4 & 2 & 2500 & $=\Pi C(\$ C \$ 9 ; A 4 ; ;-B 4)$ & $=\mathrm{D} 3+\mathrm{C} 4$ & \\
\hline 5 & 3 & 2200 & $=\Pi C(\$ C \$ 9 ; A 5 ; ;-B 5)$ & $=\mathrm{D} 4+\mathrm{C} 5$ & \\
\hline 6 & 4 & 2500 & $=\Pi C(\$ C \$ 9 ; A 6 ; ;-B 6)$ & $=\mathrm{D} 5+\mathrm{C} 6$ & \\
\hline 7 & 5 & 2500 & $=\Pi C(\$ C \$ 9 ; A 7 ; ;-B 7)$ & $=\mathrm{D} 6+\mathrm{C} 7$ & \\
\hline 8 & & & & & \\
\hline 9 & Стоимс & ость капитала & 0,2 & & \\
\hline 10 & & & & & \\
\hline 11 & Дискон & нтированный & & & \\
\hline 12 & перио & & $=A 6-D 6 / C 7$ & & \\
\hline 13 & & & & & \\
\hline 14 & 111 & Лист 4 Лис & т5 Лист6 Лист7 & T) & $\rightarrow \square$ \\
\hline
\end{tabular}

Рисунок 6 - Формулы для расчета DPP.

Следует отметить, что последнее значение столбца «Накопленный дисконтированный денежный поток» показывает чистую приведенную стоимость по проекту (которая, как известно, выступает аналогом прибыли в инвестиционном анализе). Если она известна или ее расчет производился ранее с помощью функций ЧПС или ЧИСТНЗ, то можно

ISPC Education and Innovation, 
использовать это в качестве критерия правильности произведенных расчетов [1].

Так, в нашем примере значение ячейки D7, т. е. чистая приведенная стоимость по проекту равно 302,92 тыс. руб.

Полученные значения дисконтированного периода окупаемости и чистой приведенной стоимости свидетельствуют о том, что данный инвестиционный проект эффективен (целесообразен). Срок его окупаемости (4,7 года) меньше общего срока реализации проекта (5 лет), поэтому все понесенные затраты окупятся и предприятие за оставшийся срок $(5-4,7=0,3$ года) получит небольшую прибыль (в размере 302,92 тыс. руб).

\section{Conclusion}

В заключение следует отметить, что при оценке эффективности инвестиционных проектов аналитик имеет дело с планируемыми (предполагаемыми) значениями доходов и расходов, а поскольку инвестиции характеризуются длительным периодом времени, то под воздействием внутренних и внешних факторов предполагаемые значения денежных потоков могут изменяться, что в свою очередь приведет к искажению рассчитанных на их основе критериев эффективности. Поэтому при проведении анализа следует помнить, что полученные значения дисконтированного срока окупаемости являются «примерными». На них можно ориентироваться, но не следует забывать, что они не абсолютны и могут измениться. Чем ближе $D P P$ - к общей продолжительности проекта, тем более рискован инвестиционный проект. И наоборот.

Так, в нашем примере дисконтированный срок окупаемости (4,7 года) практически равен общему сроку реализации проекта (5 лет), что предопределило невысокую величину прибыли и высокую степень риска по проекту, т. к. даже незначительное отклонение фактических значений денежного потока от запланированных может привести к появлению убытка. Запас финансовой прочности проекта практически отсутствует.

Таким образом, дисконтированный срок окупаемости можно использовать в качестве субъективного критерия в оценке проектного риска: при большом сроке окупаемости можно говорить з о значиельной степени неопределенности получения ожидаемых инвестиционных результатов, в то время как окупаемость проекта в краткосрочном периоде свидетельствует об относительно низком уровне риска.

\section{References:}

1. Poljakov VE (2016) Komp'juternye tehnologii investicionnogo analiza: ucheb. posobie / V. E. Poljakov, A. S. Kravchenko. - Krasnodar: KubGAU, 2016. - 180 p.

2. Kalmykova TS (2013) Investicionnyj analiz: ucheb. posobie / T. S. Kalmykova. - Vysshee obrazovanie: Bakalavriat. - M.: INFRA. - M, 2013. - 204 p.

3. Vahrushina MA (2011) Upravlencheskij analiz: uchebnoe posobie dlja studentov, obuchajushhihsja po special'nosti «Buhgalterskij uchet, analiz i audit»/ M. A. Vahrushina. - M.: Izd-vo «Omega-L»; 2011. $399 \mathrm{p}$.

4. (2010) Analiz investicionnoj privlekatel'nosti organizacii: nauchnoe izdanie / D. A. Endovickij, V. A. Babushkin, N. A. Baturina i dr.; pod red. D. A. Endovickogo. - M. : KNORUS, 2010. - 376 p.

5. Savickaja GV (2014) Analiz jeffektivnosti i riskov predprinimatel'skoj dejatel'nosti: metodologicheskie aspekty: monografija. - M.: Infra-M, 2014. - $272 \mathrm{p}$.

6. (2000) Metodicheskie rekomendacii po ocenke jeffektivnosti investicionnyh proektov, utverzhdennye Minjekonomiki RF, Minfinom RF, Gosstroem RF 21.06.1999 № VK 477. M.: Izd. «Jekonomika», 2000.

7. Chetyrkin EM (2011) Finansovaja matematika: uchebnik / E. M. Chetyrkin. - M.: Delo, 2011. $392 \mathrm{p}$.

8. Chernov VA (2012) Investicionnyj analiz: uchebnoe posobie / Chernov V.A. - M.: JuNITI-DANA, 2012. - 159 p.

9. Cheremnyh OS (2013) Komp'juternye tehnologii $\mathrm{v}$ investicionnom proektirovanii / Cheremnyh O. S., Cheremnyh S. V., Shirokova O. V. - M.: Finansy i statistika, 2013. - 192 p.

10. Kjehill Majkl (2012) Investicionnyj analiz i ocenka biznesa: ucheb. posobie / Majkl Kjehill. - M.: Delo i servis, 2012. -432 p. 